\title{
Casting Defects Diagnosing System Based on . NET
}

\author{
YONG Xiong ${ }^{1, a}$ \\ ${ }^{1}$ Guangdong Polytechnic, No.20 Lanshi Second Rd., Chancheng District, Foshan, Guangdong, \\ China, 528041 \\ ajacquee@126.com
}

\begin{abstract}
Keywords: defects diagnosing; expert system; .NET platform
Abstract: By comparing the existing casting defects diagnosis methods, a set of .NET-based casting defects expert system has been developed which analyzes the causes and countermeasures. By storing the defect classification knowledge, coding defects in the SQL database, and using guide form programmed in .NET platform, more precise causes and countermeasures of the casting defects can be derived. The experiment shows that the system is simple, flexible and valuable that precisely figures out the causes according to the casting defects descriptions.
\end{abstract}

\section{Introduction}

It is necessary to precisely analyze the causes of casting defects and continuously optimize the manufacturing technique that ensures the casting quality in industrial production. Defects analysis system which infers and analyzes the defects with simple structure, convenient inquiry, reasonable expression, simple maintenance and easy management plays a multiplier effect. The applications of intelligent diagnosis system facilitate casting defects analysis. Using experience and knowledge of experts who have worked in the field for many years, the intelligent defects analysis system simulates the approach to deriving defects, obtains and expresses the causes of casting defects. This paper introduces the development of a set of defects of practical reasoning system based on. NET platform, provides intuitive expression for causes of casting defects.

\section{Development Solution}

Most of existing casting defects diagnosis systems are feature-oriented which means these systems collect some types of casting defects (knowledge base), input defect characteristics (human-computer interface), present the source of casting defects and prevention and control measures based on feature-oriented information (inference engine). For example, ACDAS developed by North University of China, and FQExpert, a network defect analysis system which developed by Tsinghua University based on ICFTA defect classification. Most of these development tools based on the high-level language and database, using a high-level language to construct system framework and knowledge database to storage defects, such as $\mathrm{C}++$ and Access database[3-4], dynamic network programming language (ASP.NET) and the data server PointBase[5-6], VB, ASP.NET and Access[7], Prolog[8], etc.

Compared with other platforms, the .NET Framework supports multiple languages such as VB, C\#, ASP, etc. Applications based on .NET are relatively simple and easy to realize. As a result, this paper introduces a casting defect diagnosis expert system which is developed on .Net platform according the steps as following: (1) in the .NET platform, modifies guiding module windows using C\#, derives defect sources and suggestions in the associated windows by answering the instructions given in the guiding window; (2) Stores all kinds of casting defects, causes and countermeasures using SQL Server; (3) Reasoning machine is implemented by the guiding logic. In the process of reasoning, defects and process controls are encoded by symbols, associated with SQL sentences and guiding windows. 


\section{Design of System}

Figure 1 is the structure of the Casting Defects Diagnosis Expert System. Causes and countermeasures of defects can be educed through the human-computer interface and the kernel.

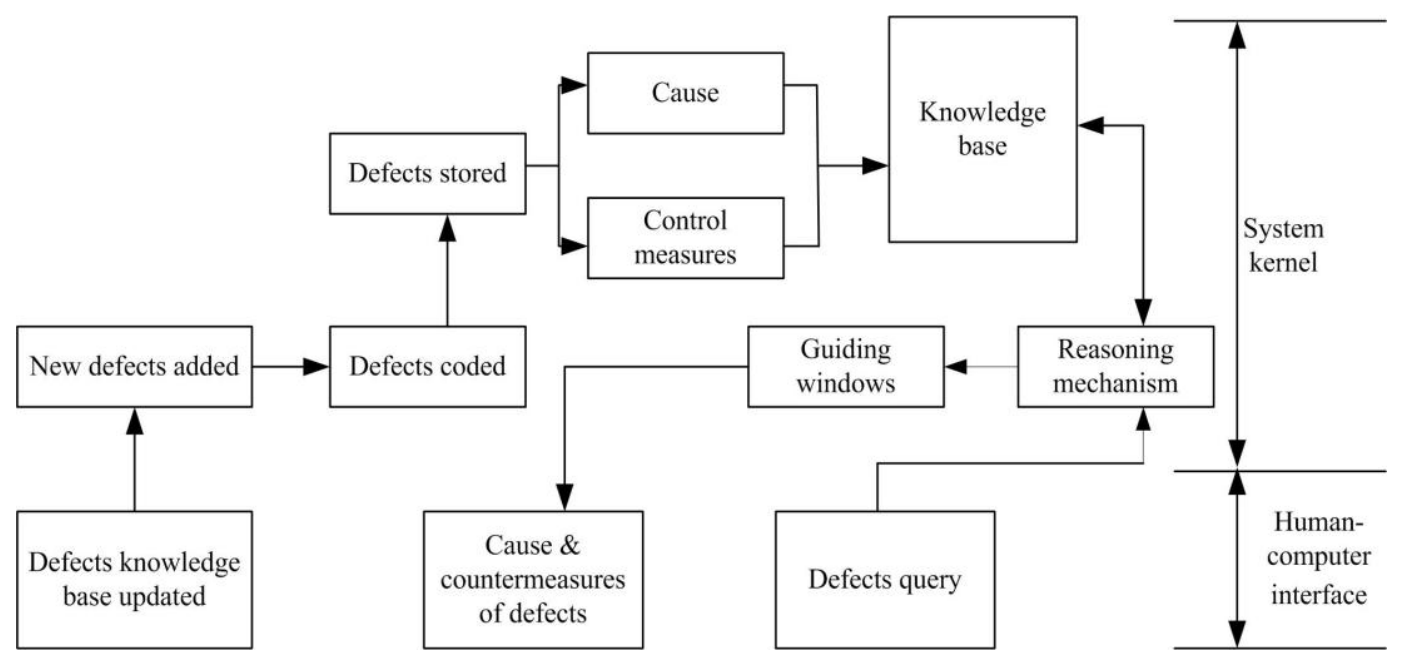

Fig.1 block framework of the system

The kernel consists of defects knowledge base and inference engine. Defects knowledge base stores causes, countermeasures and fuzzy term describing the defects which are encoded and fuzzy matched the keywords of defects; Reasoning mechanism based on the defect formation constructs guiding windows dynamically and retrieves the accumulated defect codes in the knowledge base.

Human-computer interface provides clear and concise diagnose services for users. In defects inquiry module, inputing defects characteristics, causes and countermeasures can be obtained through the guiding windows. In defects database update module, knowledge base can be updated according to causes and countermeasures by encoding and storing new defects.

\section{Defects Knowledge Base Development}

Defects knowledge base development includes coding, classification and design the structure of knowledge. Casting defects includes 8 types and 102 species as table 1 according to the method of GB/T 5611-1998 and reference [3]. Based on that, each defect is subdivided if necessary.

Tab.1 classification of defects knowledge

\begin{tabular}{clc}
\hline Code & \multicolumn{1}{c}{ Name } & Class \\
\hline A & Metallic Projection in the form of fins & 8 \\
B & Cavities & 10 \\
C & Discontinuities,Cold shuts & 9 \\
D & Defective surface & 14 \\
E & Incomplete casting & 6 \\
F & Incorrect,dimensions,shape and weight & 16 \\
G & Inclusions & 13 \\
H & Characteristic, consistance, structure abnormal & 26 \\
\hline
\end{tabular}

Knowledge structure is designed as the form of classification tree. Fig 2 is the example of classification tree of crack, cold insulation defects which causes are classified and encoded. 


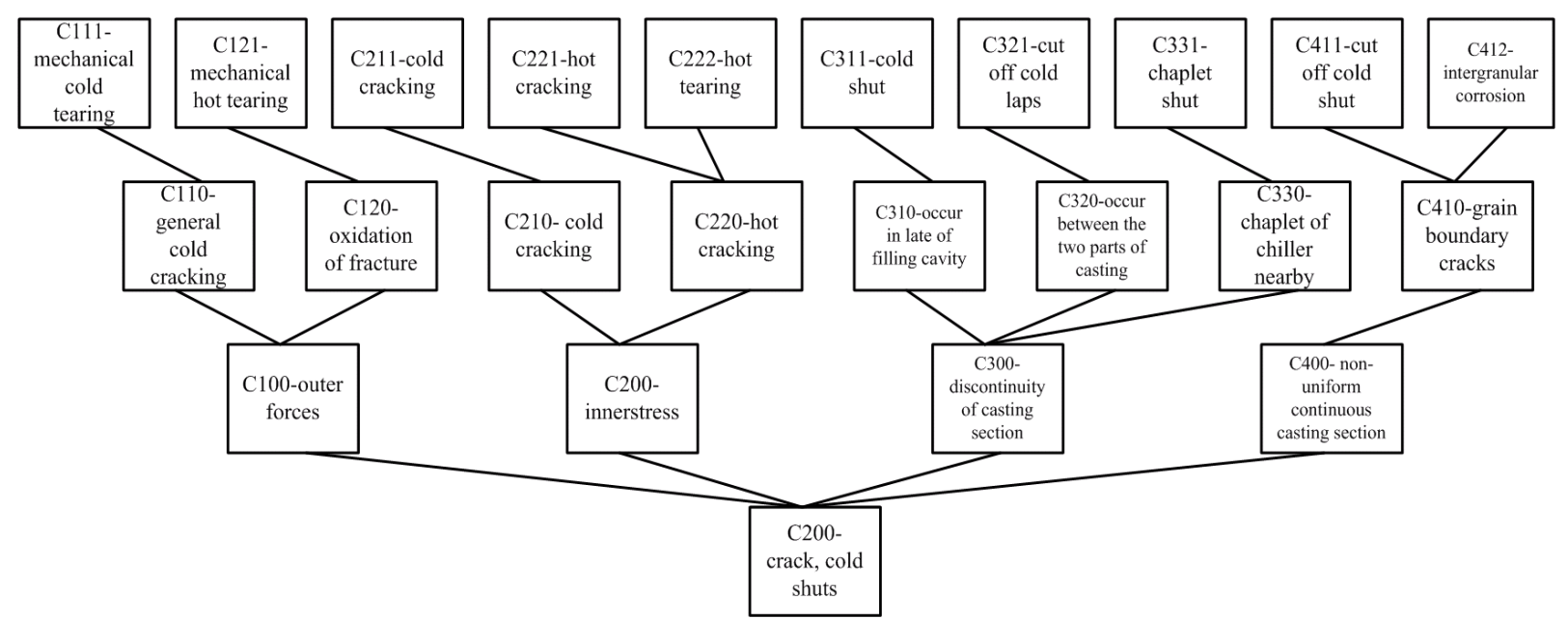

Fig.2 processing trees design of defects knowledge

Defect reasoning is implemented by defect characteristics classification in knowledge base and guiding windows as shown in fig 3. Step 1, input defect characteristics in guiding windows, reason characteristics and match defects classification in knowledge base. If the defect matched in the knowledge base still has sub branches then a new guiding window will be given. Step 2, according to the new guiding windows and judgement, the highest matching degree of defect causes and countermeasures to actual casting defect is obtained.

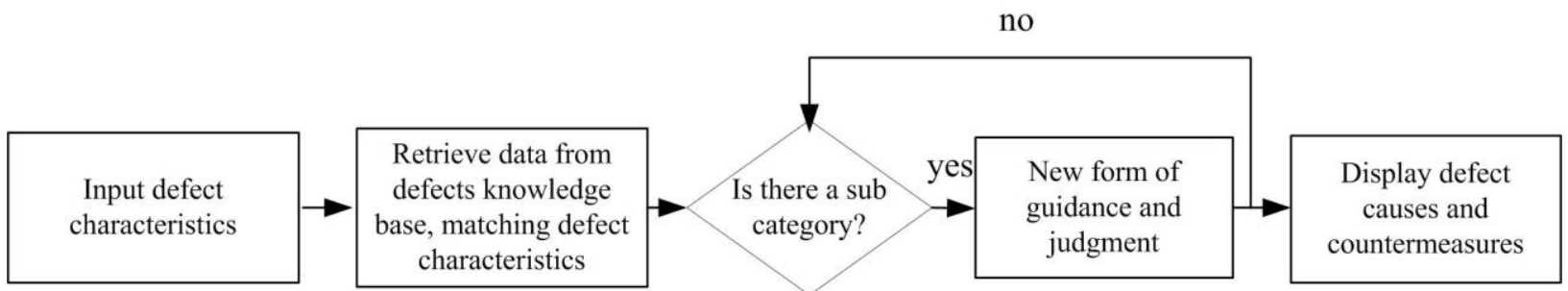

Fig. 3 defect inference flow

As shown in fig 3, the kernel of defects reasoning design is defect classification retrieval, matching and window representation. Defect classification retrieves mainly by keywords. For example, sentence such as select * from table where column like '\%CRACK\%' is programmed to retrieve crack in SQL Server. While C\# connect to SQL Server, causes and countermeasures can be expressed as string str = "insert into server(causes, countermeasures) values(' " + causesTextBox.text + "'," + countermeasuresTextBox.text + "')" in C\#.

\section{Application and Result}

A low-pressure casting aluminum alloy wheel has crack defects. To analyze its causes and countermeasures, input CRACK, LOW PRESSURE CASTING, ALUMINIUM ALLOY, WHEEL HUB in sequence, press the button of precision search (fig 4.a), window like fig 4.b is obtained. Because it is unable to identify the cold crack or hot crack, click the button of continue, a guiding window is shown as fig 4.c. The description of crack is consistent with window 4.c then press the button OK to confirm the defect of C221-stress cracking. Causes and countermeasures are given like fig 4.d.

\section{Conclusion}

The expert system which classifies, encodes and reasons the casting defects can derive causes and countermeasures of defects on-demand according to the previous defect features classification. In addition, this system is built in the platform of .NET with simple and flexible structure, easy to 
implement and maintain, which meets the general need of casting defect prevention and the promotion of quality. However, casting defects are numerous. Each type of defect has a lot of branches because of different material and casting processes. To improve the stability and reliability of the system, some efforts still need to make in two aspects. One is to create expert system for single material, single casting part and single casting technology. The other is to improve the database of casting defects classification. In a word, the casting defects diagnosis system still needs continuous efforts.

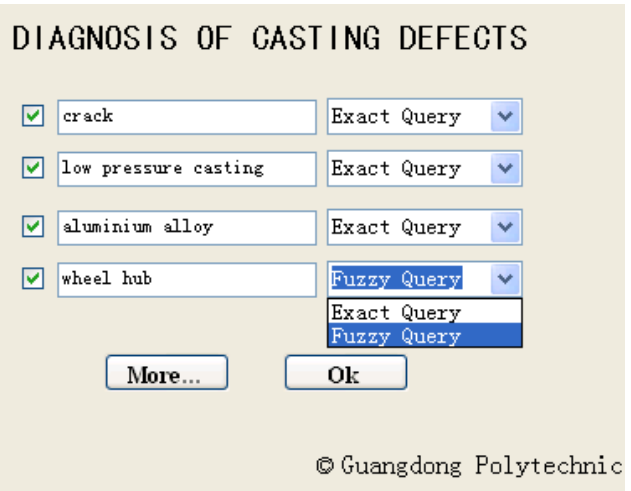

(a)

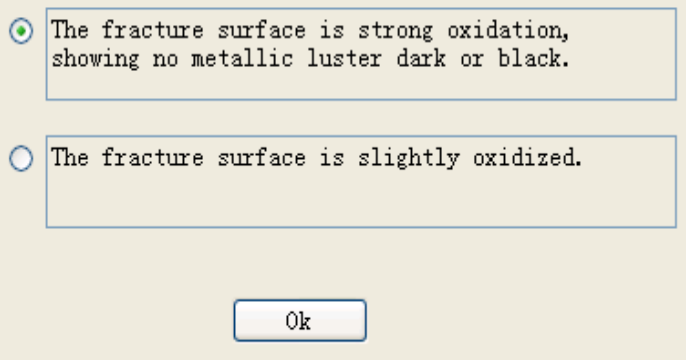

(c)

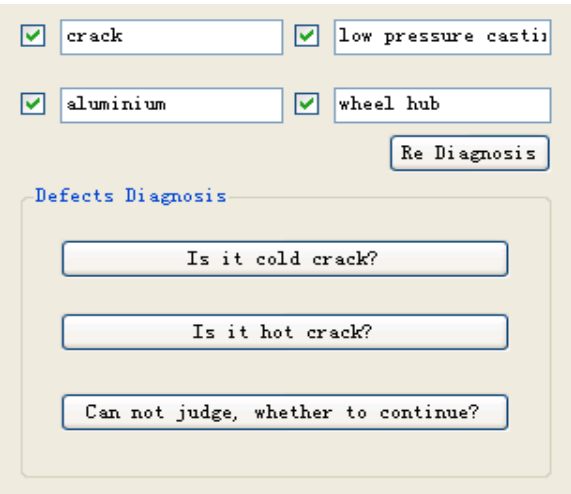

(b)

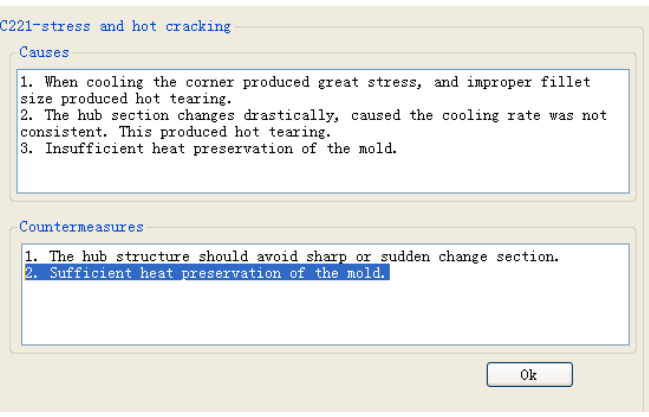

(d)

Fig.4 casting defects causes and countermeasures analysis

\section{References:}

[1] JIN Huajun and LUO Pinghui, Defects Analysis and Solving Examples of Al Alloy Casting for High Voltage Switch. Hot Working Technology, 2013. Vol.42, No.11: p.66-68.

[2] Dabade U A, Bhedasgaonkar R C. Casting defect analysis using design of Experiments (DoE) and computer aided casting simulation technique[A]. Forty Sixth CIRP Conference on Manufacturing Systems[C]. Amsterdam: Elsevier B V, 2013: 616-621.

[3] Zhang Guo-wei, Xu Hong and Mao Hong-kui, Study on Expert System Inference Analysis Control Strategy of Casting Defects in Aluminum Castings. Foundry Technology, 2011. Vol.32, No.12: p. 1690-1693.

[4] Xue Li, Xu Hong and Zhang Guo-wei, Defects Analysis and Solving Examples of Al Alloy Casting for High Voltage Switch. Foundry Technology, 2009. Vol.29, No.10: p.1344-1346.

[5] Zhang Liguang, Xiong Shoumei and Wu Junjiao, Study On Casting Defects Analysis Expert System. Chinese Journal of Mechanical Engineering, 2001. Vol.37, No.11: p.85-88.

[6] FU Zhen-nan, XIONG Shou-mei and ZHANGLi-guang, Study on Internet- based Casting Defects Analysis Expert System. Foundry Technology, 2007. Vol.28, No.1: p.118-120.

[7] LYU Bailin and Zhu Xiangzhe, Casting Defects Remote Diagnosing Expert System Based on Internet. China Foundry Machinery \& Technology, 2006(2): p.10-13. 
[8] Li ChunXiang and Chen Shengning, The Expert System For Analyzing Aluminum And Magnesium Casting Defects. Pattern Recognition And Artificial Intelligence, 1998. Vol.11, No.2: p.233-238. 